\title{
Finite Element Analysis of G-10 Flange/Board for the CC Feedthrough Box
}

\author{
Bob Wands \\ April 16,1990
}

D0 Engineering Note

3740.512-EN-247 


\author{
Finite Element Analysis of G-10 \\ Flange/Board for CC Feedthrough Box
}

Bob Wands

\title{
Introduction
}

The assembly of the stainless steel feedthrough box for the CC cryostat resulted in distortion of the slotted plates through which the circuit boards must be installed. Due to the o-ring grooves in the plates and the presence of small pump out passages it is difficult to machine the surface flat after installation. Installing the boards on the distorted surface requires distorting the $\mathrm{g}-10$ flange and the board which is glued to it to match the surface distortion and achieve a seal. This results in stresses for which the epoxy bond may not be adequate. The purpose of this work is to estimate the shear and normal stresses in the two critical planes between the board and flange and evaluate epoxy joint performance.

This work was requested by Sandor Feher, and is based on information and drawings which he supplied.

\section{G-10 Board and Flange}

The board and flange are shown in Fig. 1. The board is installed asymmetrically in the flange relative to the flange longitudinal centerline. The flange is split longitudinally on its centerline to recieve the board, then board and flange are epoxied into a single unit.

The direction and assumed shape of the required distortion are indicated in the figure. This distortion is based on surveyed measurements taken on the steel plates. 


\section{The Finite Element Model}

The finite element model is shown in Fig. 2. The model assumes bilateral symmetry. The ANSYS STIF 45 8-node solid element is used in most of the model, although it is allowed to degenerate to a wedge shape where necessary for conforming to the curved end. The nominal length of an element side is 0.25 in.

The loading was input as specified nodal displacements along the two areas shown. A circular distorted shape with a maximum displacement of 0.012 inches at the board center was assumed, and a prep7 macro was used to calculate the required displacement based on the nodal $x$-coordinate. Symmetry boundary conditions were used at $x=0$. The termination of the board in $z$ was arbitrarily taken as approximately 4 inches on either side of the flange. This was assumed to be far enough to represent the extreme stiffness of the board to bending about the $y$-axis.

\section{$\underline{\text { Results }}$}

\section{Distorted Structure}

Fig. 3 shows the flange/board distortion, and verifies that the shape is input correctly. The distortion of the flange at the board centerline in the end view shows the flange bending to achieve the required displacement. This occurs because of the very large bending stiffness of the board, which does not allow it to distort significantly.

\section{Shear Stresses}

The shear stresses in planes A-A and B-B (on either side of the board) are shown in Figs. 4 and 5. A maximum working shear stress for the epoxy bond was specified by Sandor Feher as 1000 psi. In the Fig. 4a and 5a, the portion of each plane in which the shear stress exceeds 1000 psi is indicated. Figs. 4b and $5 \mathrm{~b}$ show the rapidly changing shear stress state near the end of the flange. The maximum shear stress in each plane is at the end of the flange, as would be expected from an analogy with a simply supported beam loaded along it's span. Such a beam has its maximum shear force at the supports. 
The stress plots show concentrations associated with geometric discontinuities at the ends. This model does not have sufficient detail to calculate such concentrations accurately, since to do so would require accurate modeling of fillets in both glue and flange, and considerable mesh refinement about these fillets. The most salient features of the stress plots are the extent of shear stresses in excess of 1000 psi away from discontinuities, which are best shown in Figs. $4 a$ and $5 \mathrm{a}$.

\section{Normal Stresses}

The normal stresses in planes A-A and B-B are shown in Figs. 6 and 7 . If the epoxy behaves as a brittle material, then compressive normal stress has little effect on performance. Although a maximum working tensile stress was not specified, it might be assumed that a valid maximum is twice the working shear stress, or 2000 psi. Figs. $6 a$ and 7 a show the extent of tensile stress exceeding $2000 \mathrm{psi}$ in the two planes. Figs. $6 \mathrm{~b}$ and $7 \mathrm{~b}$ show the stress distribution near the flange end in more detail.

\section{Comments on Model Accuracy}

Each element of a finite element model calculates stresses for each of it's nodes. Depending on mesh density, every element connected to a given node will calculate a different stress for that node. During the post-processing, the results at a node may be averaged for any or all of the elements connected to the node. The ANSYS program gives the user complete flexibility in deciding which elements to include in such an averaging. In this work, there are in general three choices of elements to include in presenting the stresses calculated in the two planes. Namely, the board elements alone, the flange elements alone, or all elements together. It was found that, for the board to flange joint, the board elements gave the most pessimistic stressese, while the flange elements gave the most optimistic. The combination of the two was inbetween. The stresses here are averaged for the combination of board and flange elements. It should be noted that even the most optimistic stress presentation included substantial areas above the 1000 psi working shear stress value.

A coarse model using a nominal element size of 0.5 in. was run to check discretization error. Unfortunately, this model did not coarsen the mesh by the factor of 2 as might normally occur, since mesh density is also a function of the size of projections and grooves, which are smaller that the 0.5 in in most cases. However, some coarsening did result, and a comparison of the stresses with the fine mesh showed excellent agreement over the bulk of the two critical planes, with the coarse mesh predicting slightly smaller peak stresses at the concentrations, as would be expected. 


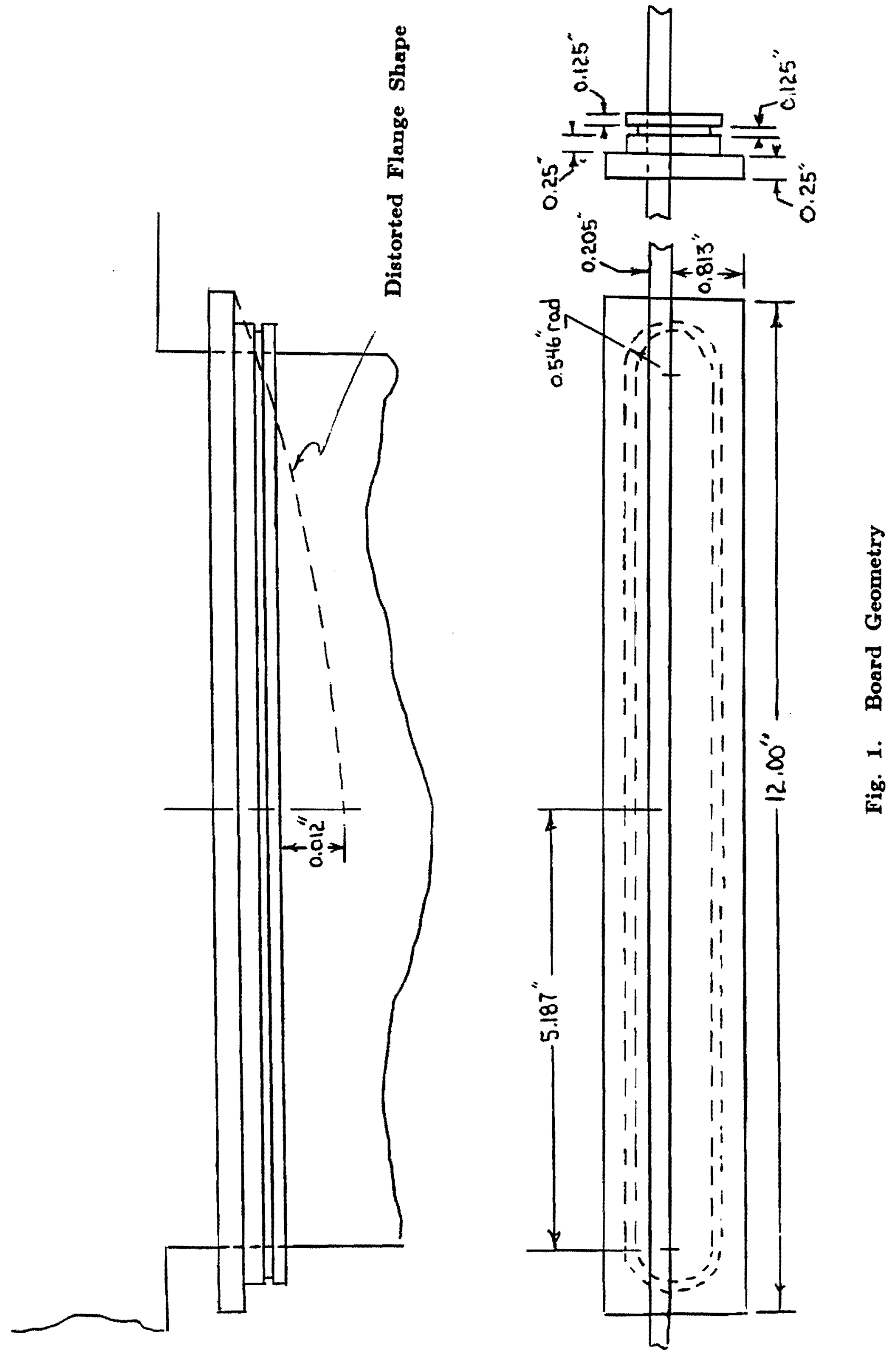




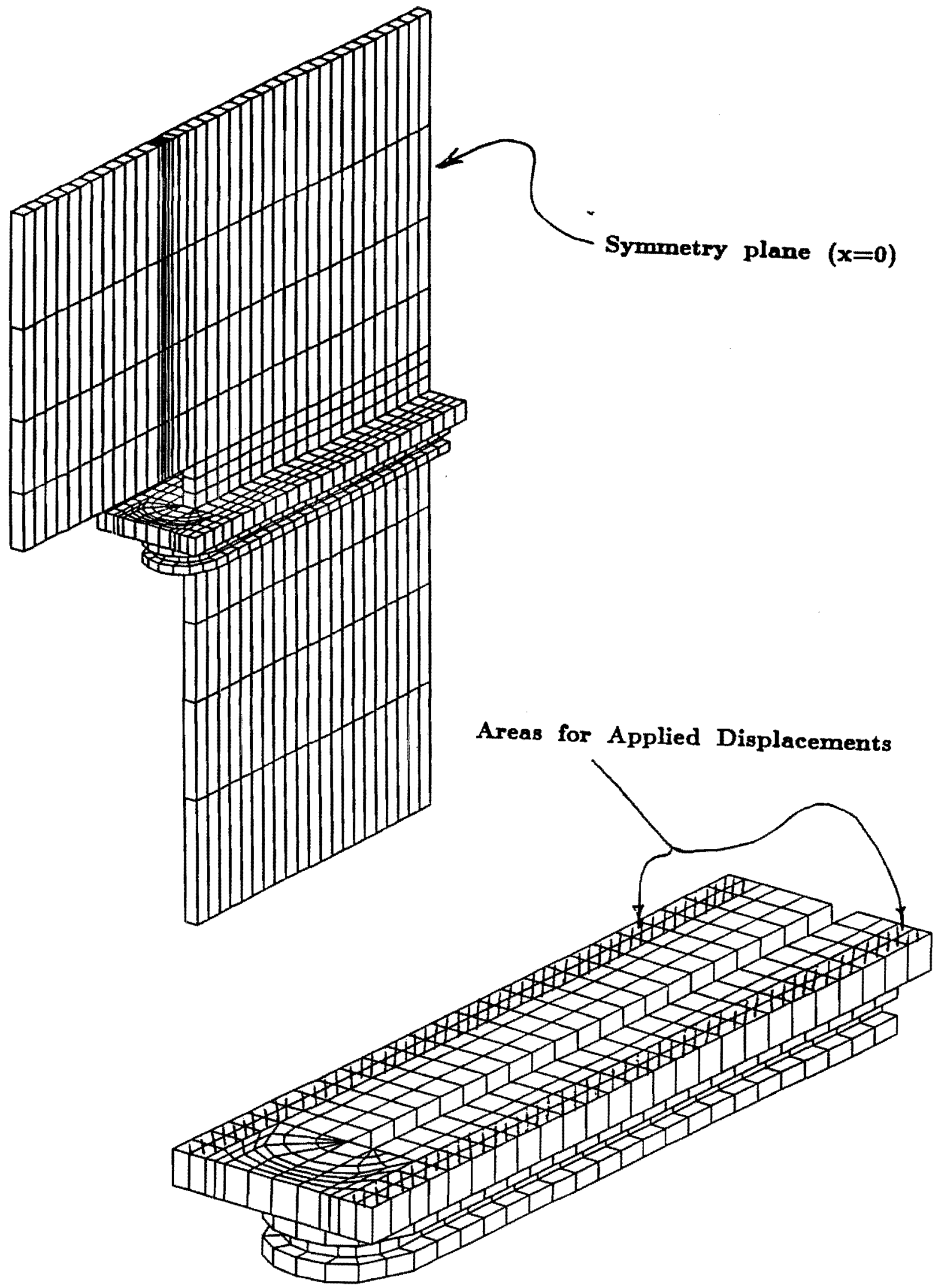

Fig. 2. Finite Element Model 

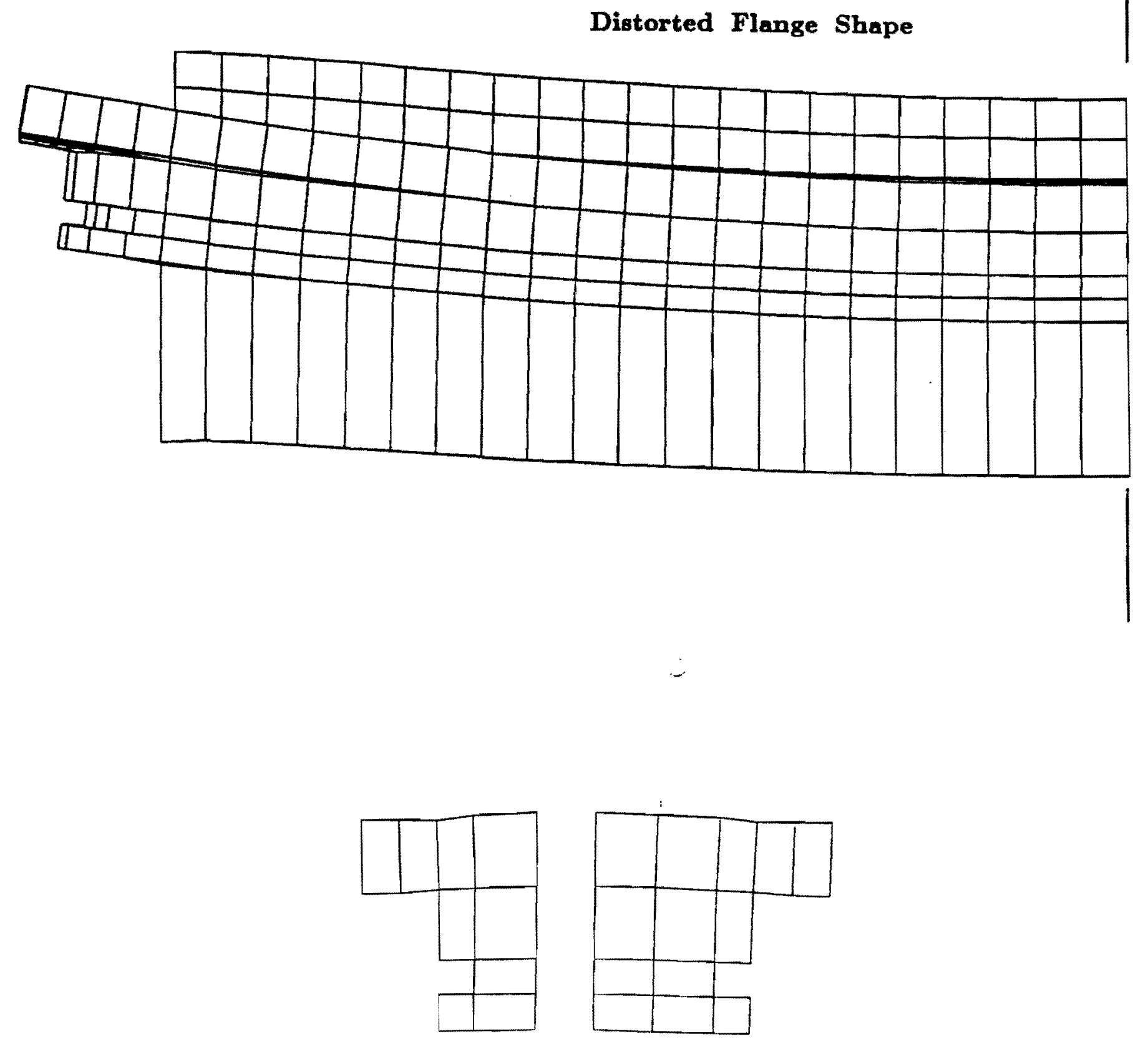

Flange distortion at symmetry plane

Fig. 3. 


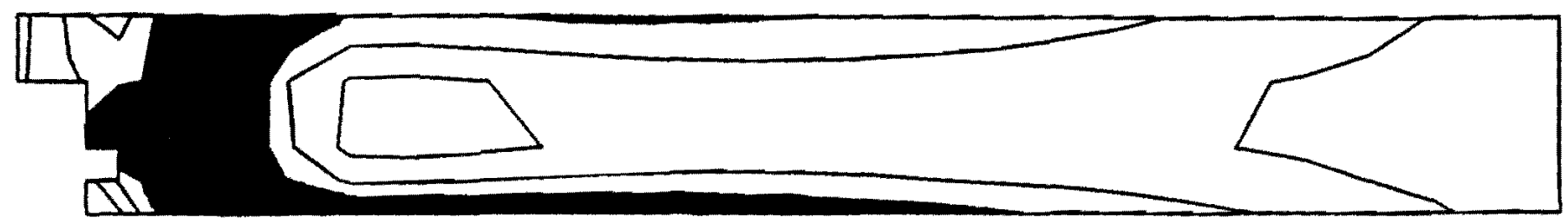

4a. Shaded area above 1000 psi shear.

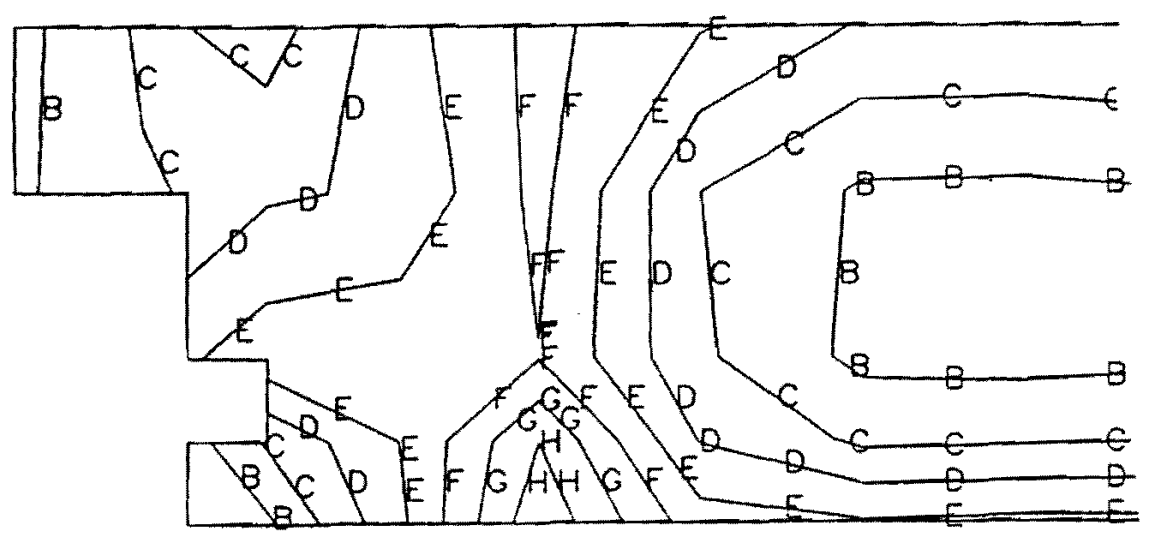

$$
\begin{aligned}
& A=-167 \text { psi } \\
& B=195 \\
& C=\mathbf{5 5 8} \\
& D=020 \\
& E=1283 \\
& F=1646 \\
& G=2008 \\
& H=2370 \\
& I=2733
\end{aligned}
$$

4b. Detailed shear stress at end

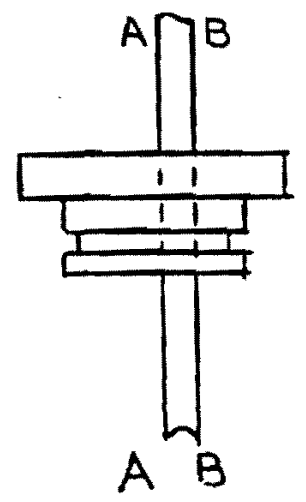

Fig. 4. Shear Stresses in Plane A-A 


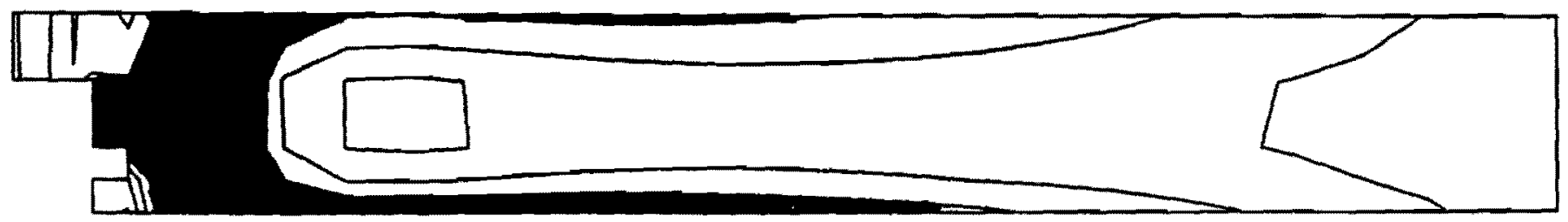

5a. Shaded area above 1000 psi shear

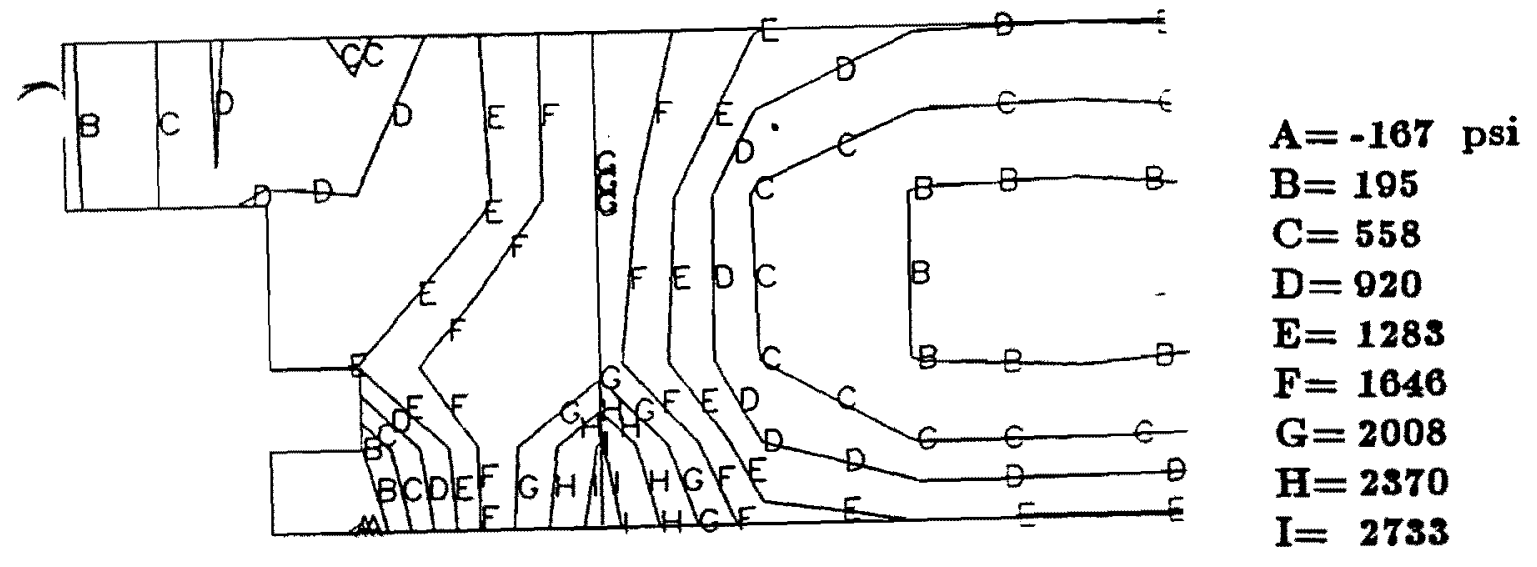

5b. Detailed shear stress at end

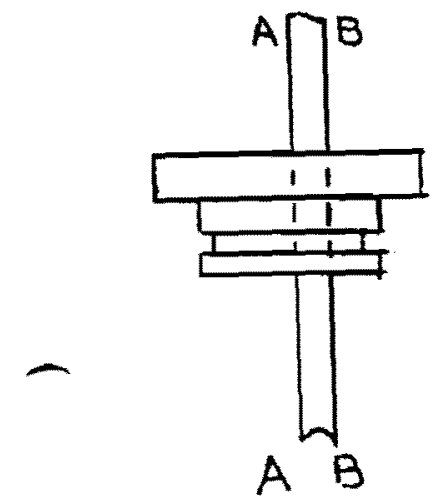

Fig. 5. Shear Stresses in Plane B-B 


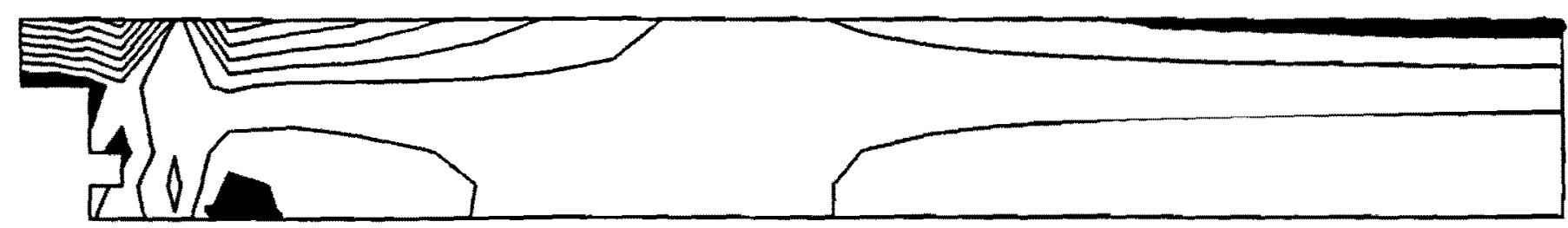

6a. Shaded area above 2000 psi tension

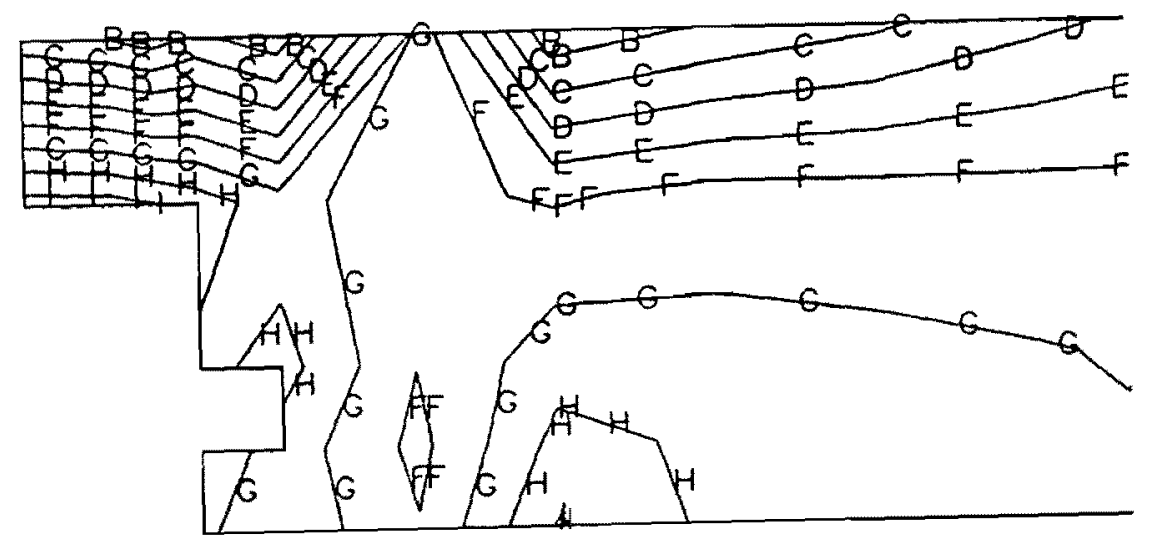

$A=-6866$

$B=\mathbf{- 5 5 6 2}$

$C=-4259$

$\mathrm{D}=\mathbf{- 2 9 5 6}$

$E=-1653$

$\mathrm{F}=\mathbf{- 3 5 0}$

$\mathrm{G}=\mathbf{9 5 3}$

$\mathrm{H}=\mathbf{2 2 5 6}$

$I=\mathbf{3 5 6 0}$

6b. Detailed normal stress at end

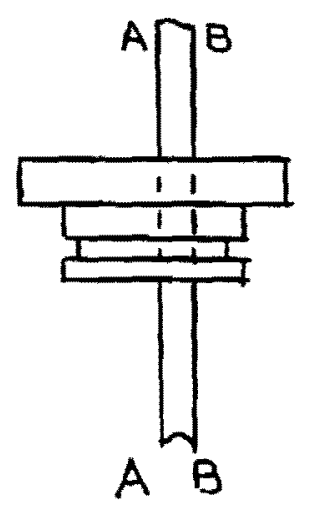

Fig. 6. Normal Stresses in Plane A-A 


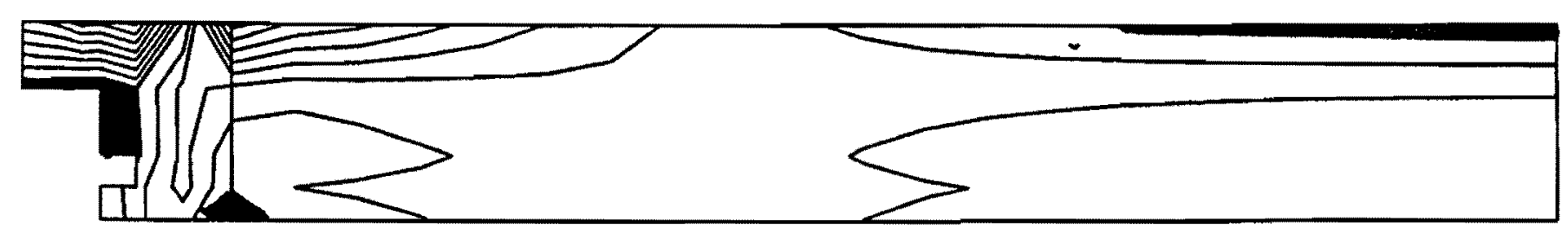

7a. Shaded area above 2000 psi tension

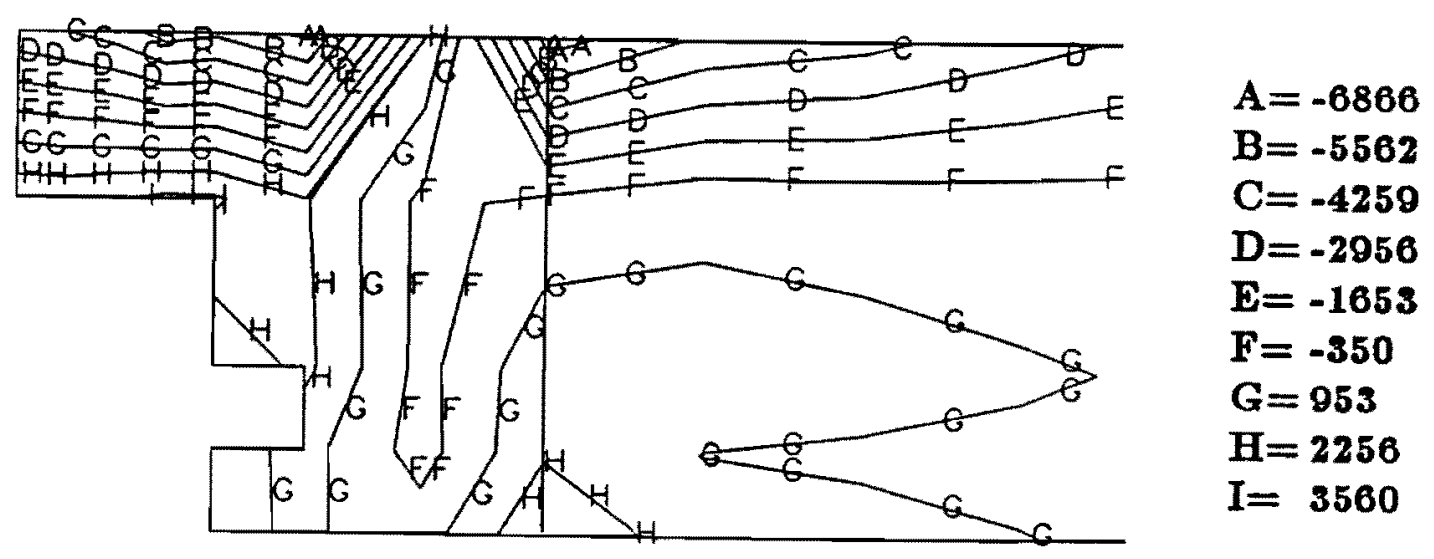

7b. Detailed normal stress at end

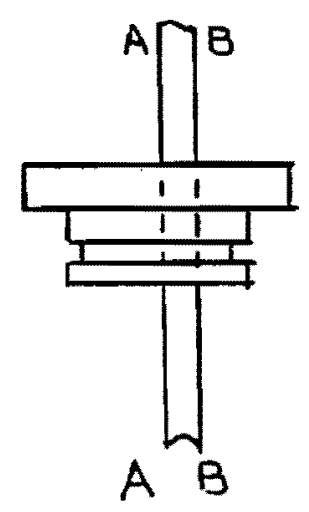

Fig. 7. Normal Stresses in Plane B-B 